九州大学学術情報リポジトリ

Kyushu University Institutional Repository

\title{
Computational Study on Femoral Fracture using CT-Image based Finite Element Method
}

\section{Zaw Linn Htun}

Interdisciplinary Graduate School of Engineering Sciences, Kyushu University

Todo, Mitsugu

Interdisciplinary Graduate School of Engineering Sciences, Kyushu University

https://doi.org/10.5109/4738567

出版情報: Proceedings of International Exchange and Innovation Conference on Engineering \& Sciences (IEICES).7，pp.58-63，2021-10-21. 九州大学大学院総合理工学府 バージョン：

権利関係: 


\title{
Computational Study on Femoral Fracture using CT-Image based Finite Element Method
}

\author{
Zaw Linn Htun ${ }^{1,3}$, Mitsugu Todo ${ }^{1,2^{*}}$ \\ ${ }^{1}$ Interdisciplinary Graduate School of Engineering Sciences, Kyushu University, Kasuga 816-8580, Japan. \\ ${ }^{2}$ Research Institute for Applied Mechanics, Kyushu University, 6-1 Kasuga-koen, Kasuga 816-8580, Japan. \\ ${ }^{3}$ Department of Physics, University of Yangon, 11041 Kamayut, Yangon, Myanmar. \\ ${ }^{*}$ Corresponding author email: todo@riam.kyushu-u.ac.jp
}

\begin{abstract}
The risk of hip fractures in osteoarthritis patients has increased due to bone structure deterioration and increased incidental falls. This study aimed to determine the strength and fracture location of the normal and osteoarthritis femurs. The quantitative computed tomography (QCT) based finite element method (FEM) was used for the analysis. Threedimensional models were constructed and simulated through Mechanical Finder. Bone mineral density (BMD) was estimated and considered as one of bone material properties index. Young's modulus (E) and strain energy density (SED) distribution were also investigated. A strong correlation between strength and average BMD was observed in normal femurs. In the osteoarthritis femur, the fracture may occur in both the head and neck region, whereas the neck region is mostly fractured in normal femurs. The average femoral strength of the normal femurs was found to be approximately $3957 \pm 293 \mathrm{~N}$, while $3549 \pm 180 \mathrm{~N}$ is for that of osteoarthritic ones.
\end{abstract}

Keywords: Finite element method; Bone mineral density; Femoral strength; Failure element; Strain energy density

\section{INTRODUCTION}

Osteoarthritis (OA) and hip fractures are common musculoskeletal disorders, and have considerable impact on the individual patient, resulting in pain and/or disability on society. Therefore, the economic burden on patients and society is considerable. With hip fracture incidence increasing, the study of optimum strengths and fracture site estimation would be very useful to predict the risk of hip fracture and monitor the effects of bone disease therapies. To estimate fracture strength of the bone, finite element method (FEM) is an effective tool that can simulate stress distribution of the bone structure $[1,2]$. It is a noninvasive method and more accurately predict the femoral strength than other methods such as bone densitometry and other diagnostic imaging's. Along with computed tomography (CT), FEA can evaluate various bone aspects, including bone geometry, cortical and trabecular bone distribution, and loading direction as well [3]. With this advantage, FEM was applied in this study to predict the proximal femoral strengths and potential fracture sites of the normal and osteoarthritis femurs.

\section{MATERIALS AND METHODS}

The femoral CT data of 20 patients (19 women aged 52.05-83.11 years, the average age of 67.63 years; and one man, age of 74.11 years) were collected from Saga university hospital, Saga prefecture, Japan. Mechanical Finder v.10 (Research Center of Computational Mechanics Inc., Tokyo, Japan) was used to generate the finite elements (FE), femoral models. 3D imaging, meshing, material sorting and boundary condition were set, and the constructed models were simulated afterwards.

\subsection{Nonlinear Finite Element Analysis (FEA)}

FE models were generated based on the surface mesh of the model and fill the internal model with the tetrahedral elements. The elements were manually set with a minimum size of $1 \mathrm{~mm}$ and a maximum of $2 \mathrm{~mm}$ to get a finer model as illustrated in Fig.1 [4]. Moreover, the inhomogeneous bone models were developed other than homogeneous as there was some limitation with homogeneous model reported by other researchers [5-7]. Because of the structural complexity of the proximal femur, tetrahedral elements instead of cubic elements have adapted to represent the smooth surface [8]. After setting the material properties of the solid elements, 2D triangular shell elements with a thickness of $0.3 \mathrm{~mm}$, were set on the outer surface of the cortex to accurately represent the thin cortical shell [9]. Shell elements were used to simulate a cortical bone and strengthen its thinner part, and as well as to obtain an accurate evaluation of analytical value, such as stress and strain, on the model surface.

Young's modulus and yield stress of each tetrahedral element were calculated using the methods proposed by Keyak (1998) [3]. Poisson's ratio of 0.4 was also set for each element. The Drucker-Prager criterion was applied to determine the yielding, and the maximum principal stress and the minimum principal strain criteria for element failure in both tension and compression. Yield in compression was determined as occurring when Drucker-Prager equivalent stress exceeded element yield stress. Element failure in compression was then decided as occurring when the negative value of maximum principal strain exceeded 10,000 crush strain (micro strain). Bone strength or fracture load was defined as the load when at least 15 shell elements failed. In this study, shell termination was set to 15 for cracked and crushed elements.

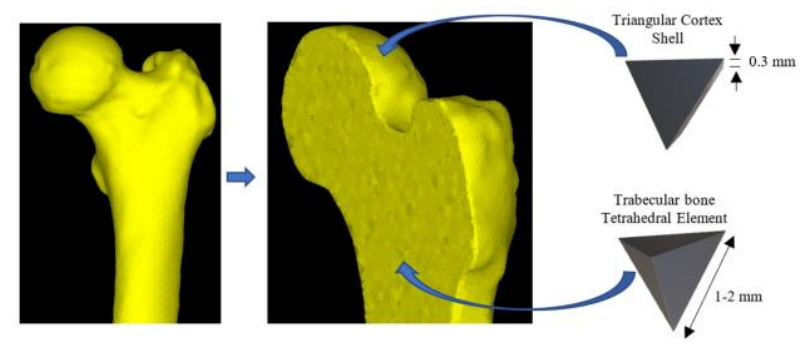

Fig. 1: FE model consisting of trabecular bone and the inner portion of cortical bone, as well as the outer cortical shell. 


\subsection{Loading and Boundary Condition}

The bone axis was set by the femoral method (Fig.2a), and the default reference femur pattern was used (Fig.2b) The constraint was assigned approximately $15 \mathrm{~mm}$ below the lesser trochanter to the base of the femur (Fig.2c). After setting this restriction, a total load of $10000 \mathrm{~N}$ was applied to the model under stance configuration (Fig.2d). Because of non-linearly fracture line prediction, the total load was divided into 10 steps followed by 4 sub-steps (i.e. 40 total output steps). In the loading direction, $\alpha$ is defined as degrees tilt from the axis while $\beta$ was the degrees turn around the axis (Fig.2e) [10].
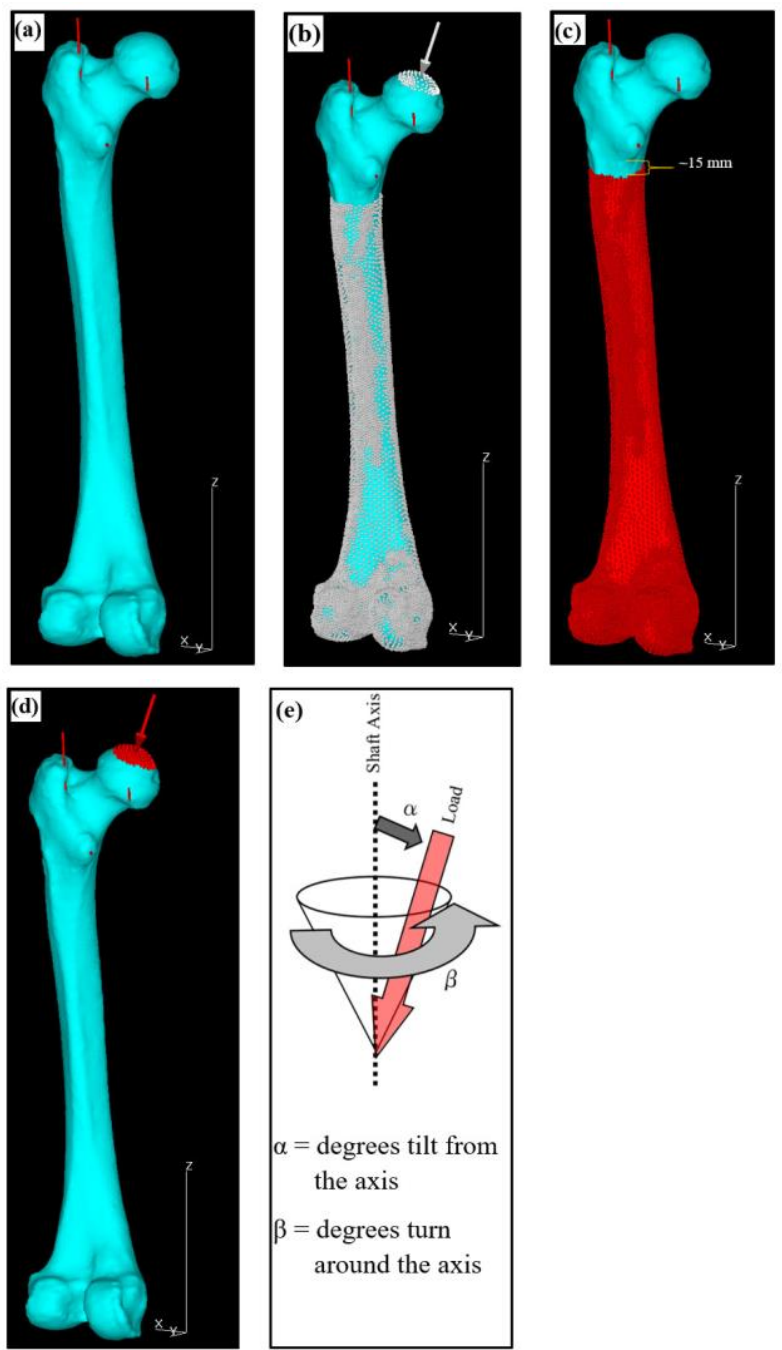

Fig. 2: Constraint and loading conditions description.

\subsection{Specification of Femoral Model}

The normal and osteoarthritis femurs were distinguished based on the patient's clinical data. The 3D FE models of normal and osteoarthritis femurs are presented in Fig. 3. The femurs with an implant and completely deformed head were not considered to account. The bone mineral density was calculated and considered as one of the bone's materials properties indices.

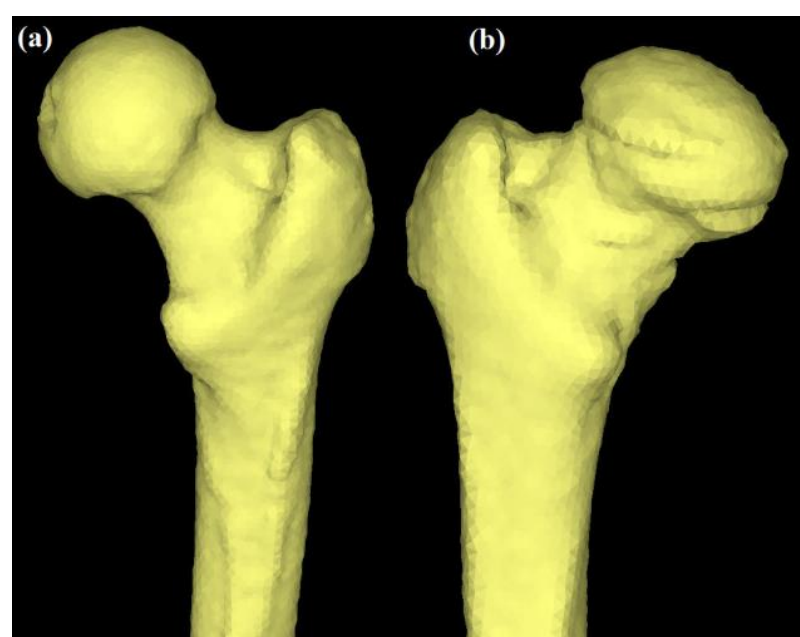

Fig.3: 3D FE model of the normal femur (a), and osteoarthritis femur (b).

\section{RESULTS}

\subsection{Femoral Strength and BMD Correlation}

The BMD is an important parameter for predicting fracture load, and in this study, the femoral head and neck region BMDs of the normal femurs were ranged from 0.20 to $0.34 \mathrm{mg} / \mathrm{mm}^{3}$. For osteoarthritis, it ranged from 0.20 to $0.43 \mathrm{mg} / \mathrm{mm}^{3}$. However, the estimated femoral strengths of the former are significantly higher than the latter. The correlation between fracture load and BMD is presented in Fig.4, and it is obvious that the femur with more femoral head density can bear the higher fracture load in both groups. A better correlation coefficient of $\mathrm{R}=0.90$ was found in the normal femurs compared to osteoarthritis femurs of $R=0.82$. The average strength of the normal femurs was found to be $3957 \pm 293 \mathrm{~N}$ (range from 2825 to 5625 ), while $3549 \pm 180 \mathrm{~N}$ (range from 1975 to 4825 ) is for that of osteoarthritic ones. At here, the \pm sign referred to standard error.

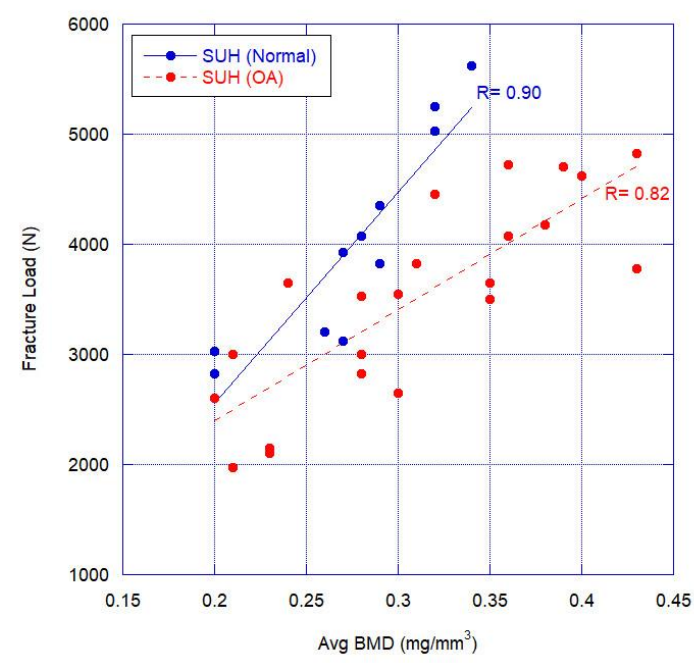

Fig.4: Correlation between fracture load and BMD 


\subsection{Comparison between Normal and Osteoarthritis Femurs}

In this section, a comparison between a normal and an osteoarthritis femur from each category, both of which showed almost the same density value of $0.32 \mathrm{mg} / \mathrm{mm}^{3}$, will be presented.

\subsubsection{BMD and Young's Modulus Distribution}

The BMD distribution patterns of described femurs are illustrated in Fig.5. From the figure, the density is fairly distributed in the normal femur, especially, in the head and neck region. Likewise, a better Young's modulus (Elastic Modulus, E) distribution than the osteoarthritic femur was also observed, as shown in Fig.6. Therefore, the normal femur can have more ability to resist the applied load.

On the other hand, a total of 190893 elements are involved in the head and neck region of the normal femur Which of them, 167472 elements appeared to be highdensity elements. Whereas osteoarthritis consists of 133074 elements, and 103808 represent high-density. In this study, the elements with a density lower than 0.13 $\mathrm{mg} / \mathrm{mm}^{3}$ which is the weakest femur's density, were considered as low-density elements, and density higher than or equal to $0.13 \mathrm{mg} / \mathrm{mm}^{3}$ were considered as highdensity elements. Due to a high number of elements as well as high-density elements, bone porosity or the amount of bone volume filled with pores or cavities might reduce in the normal femur, especially in the trabecular region. It affects the strength of the bone and therefore, the normal femur appears to be stronger ( 5250 $\mathrm{N})$ than that of osteoporosis $(4450 \mathrm{~N})$.

The coronal cross-section (medial-lateral plane) pictures of Fig.5 and Fig:6 confirm that the normal femur possesses a better stiffness compared to osteoarthritic one, and more stress will be needed to create the same amount of strain. The lesser cortical bone thickness is also noticed in the osteoarthritis femur than the normal one. This fact has a consistency with previous research, in which low-risk femurs have a larger cortical bone thickness and a higher cortical index than high-risk femurs [11].

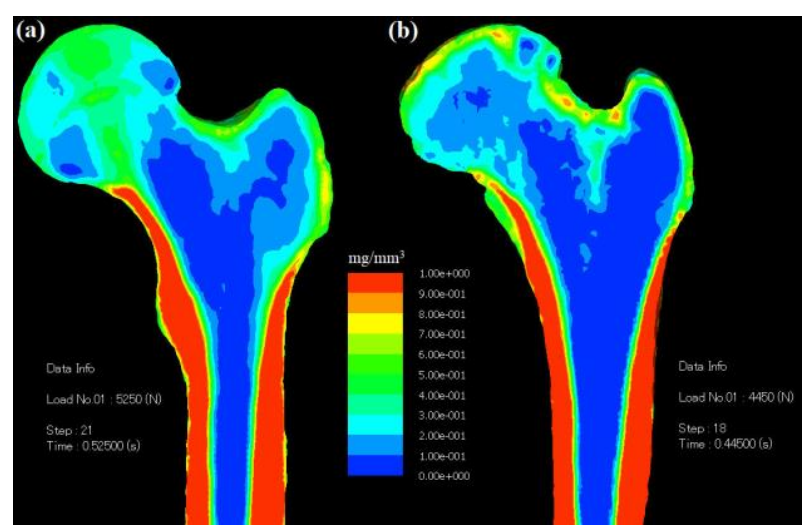

Fig.5: Density distribution of the normal femur (a), and osteoarthritis femur (b).

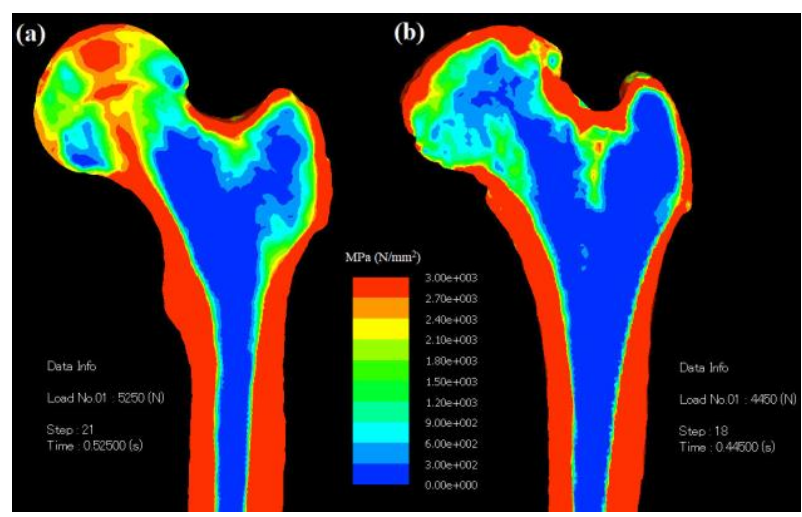

Fig.6: Young's modulus distribution of the normal femur (a), and osteoarthritis femur (b).

\subsubsection{Strain Energy Density Contour}

The strain energy density (SED) is also one of the mechanical properties which has been studied to examine their role in the regulation of BMD distribution. Fig.7 shows the SED's contours of the above two bones from each category, and it reveals that the normal femur has an average SED of $4.96 \mathrm{E}-03 \mathrm{KJ} / \mathrm{m}^{3}$, in which the SED distributes in the head and neck as well as subtrochanteric regions. Whereas, the osteoarthritis femur shows a lower SED of $4.71 \mathrm{E}-03 \mathrm{KJ} / \mathrm{m}^{3}$, and it distributes mainly in the head and neck region. As the SED is basically the product of stress and strain, a larger SED means that the more energy will store in the elements, and consequently turn into a stiffer bone.

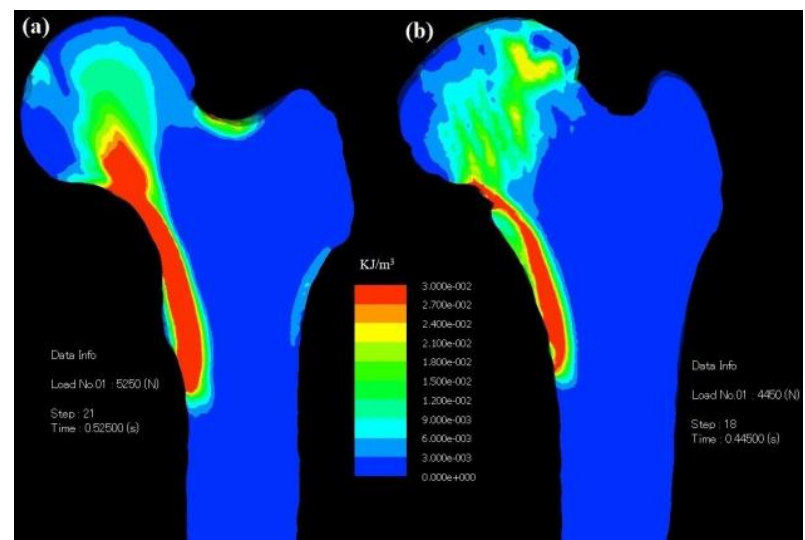

Fig.7: SED distribution of the normal femur (a), and osteoarthritis femur (b).

\subsubsection{Failure Elements Analysis}

In the fracture process, the element becomes plastic when equivalent stress exceeds yield stress, and when the minimum principal strain of plastic element exceeds crush strain (in other words, when the specified strain occurs in the compression direction), it becomes compression failure (crush). Whereas, tensile failure (crack) will happen when the principal stress exceeds the critical stress. A solid element is considered to be cracked when cracks occur in one of its three directions.

The total failure elements (crack, plastic and crush) of the normal and osteoarthritis femurs are shown in Fig.8, and it reveals that there is no compression (only tensile) failure in normal femur while both tensile and compression failures occur in osteoarthritic ones. This fact implies that the normal femur yields a lower compression strain over the abnormal one. 

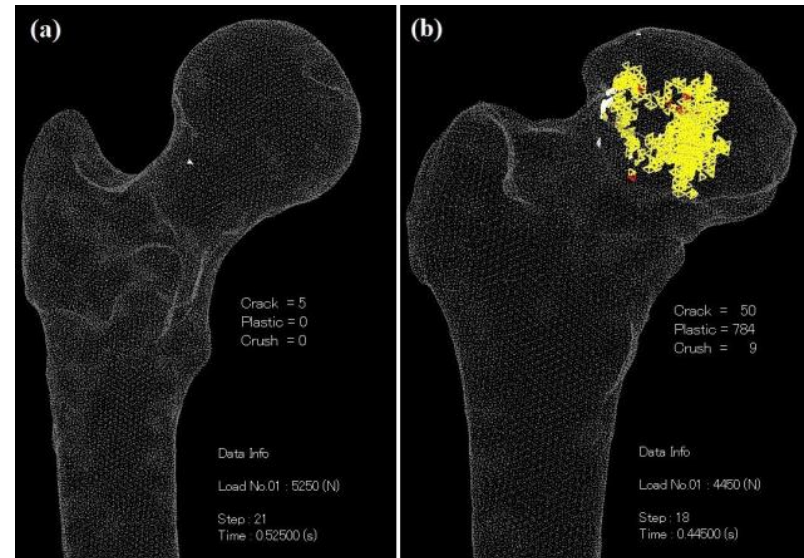

Fig.8: Failure elements accumulation of the normal femur (a), and osteoarthritis femur (b).

\subsubsection{Step-by-Step Load and Elements Accumulation}

To analyze non-linearly fracture line prediction, a substep loading system in which there are 40 total output load steps, was performed. The load steps of the simulated femoral model attribute a total number of failure elements as well as the ability of bone's resistance. Fig.9 shows the failure element increment in terms of load steps between the normal and osteoarthritis femurs. In the former, a load step elevation up to 21 can be achieved and merely 5 elements were failed. In the latter, however, the maximum level it can bear is only 18 steps, and a total of 1852 elements were fracture. Therefore, it can be realized that the increase in load step implies the stronger of the bone, and a significantly high number of failure elements can occur in a weaker bone. The strength of the femur is actually the final load at the maximum step because the elements in the model will fail when the applied stress reaches the maximum strain limit of each femur.

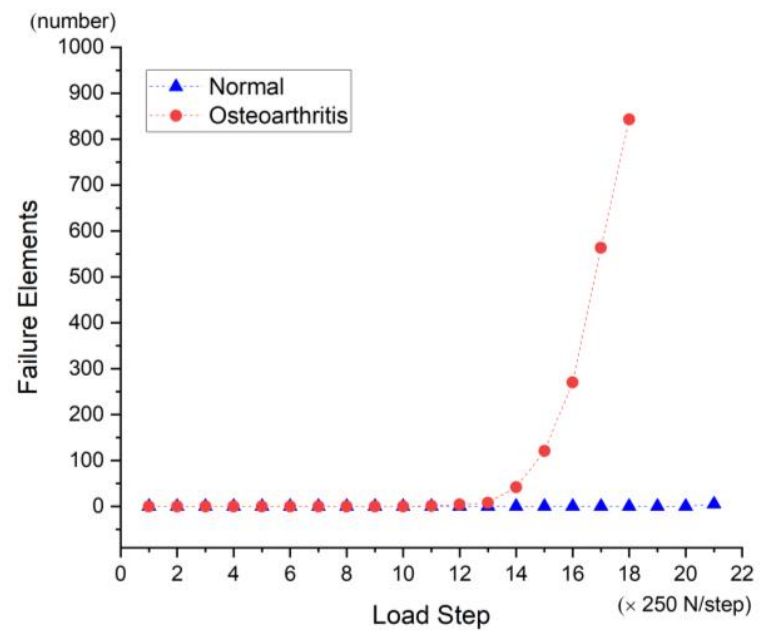

Fig.9: Failure elements increasement with respect to the load steps.

\subsection{Estimation of Fracture Location}

To estimate the fracture locations, the fracture sites of all normal and osteoarthritis femurs were thoroughly investigated. According to failure element analysis, the fracture sites were estimated in the intracapsular region in general, for both normal and osteoarthritis femurs. The fracture locations of different normal femurs are displayed in Fig.10, and that of osteoarthritis are in Fig.11, respectively.

From Fig.10, it could be understood that the normal femur might fracture on the neck region although there is little possibility on the head region too, and a plenty of failure elements accumulation can be seen on the neckcapital area of the neck region. There is also a possibility of fracture on transcervical and basicervical area of the neck region. However, it could be related to tensile fracture only because the crack elements were mostly failed in this area.
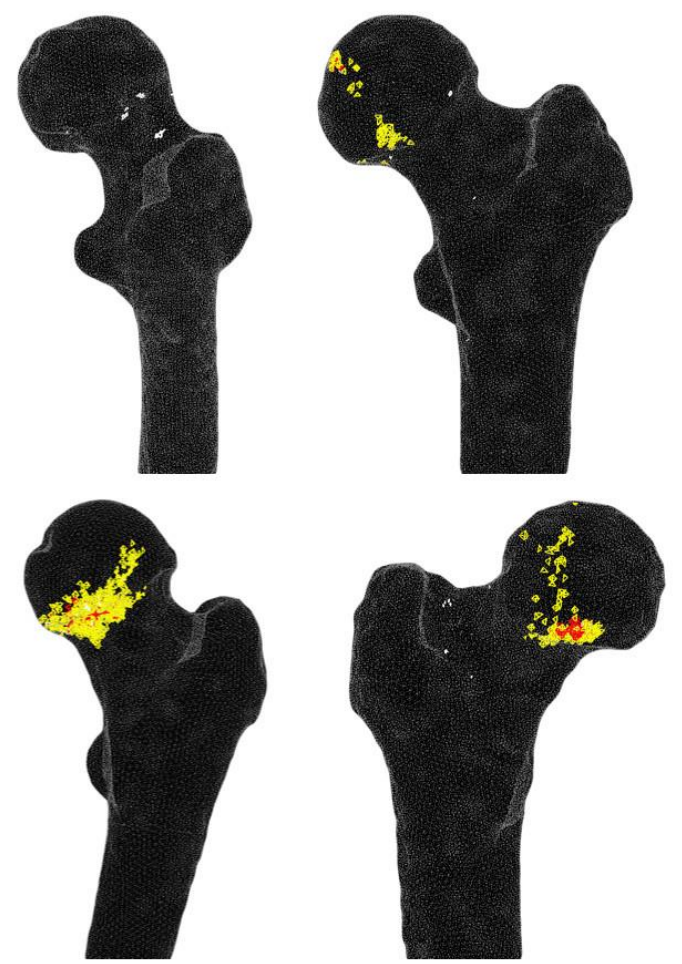

Fig.10: Estimated fracture locations of different normal femurs.

Some characteristics like fracture in the neck region were also observed in osteoarthritis femurs, as shown in Fig. 11. Nevertheless, a high possibility of fracture in the head and neck region was occurred compared to the normal ones. Higher risk in the head region could be due to femoroacetabular impingement (FAI). The total number of failure elements are also apparently more than the normal femurs. In contrast to the normal ones, a low possibility of fracture in the trochanteric region was observed in 1 out of 22 osteoarthritis femurs. As the crack elements have only appeared in that region, the fracture type might be tensile as well. 

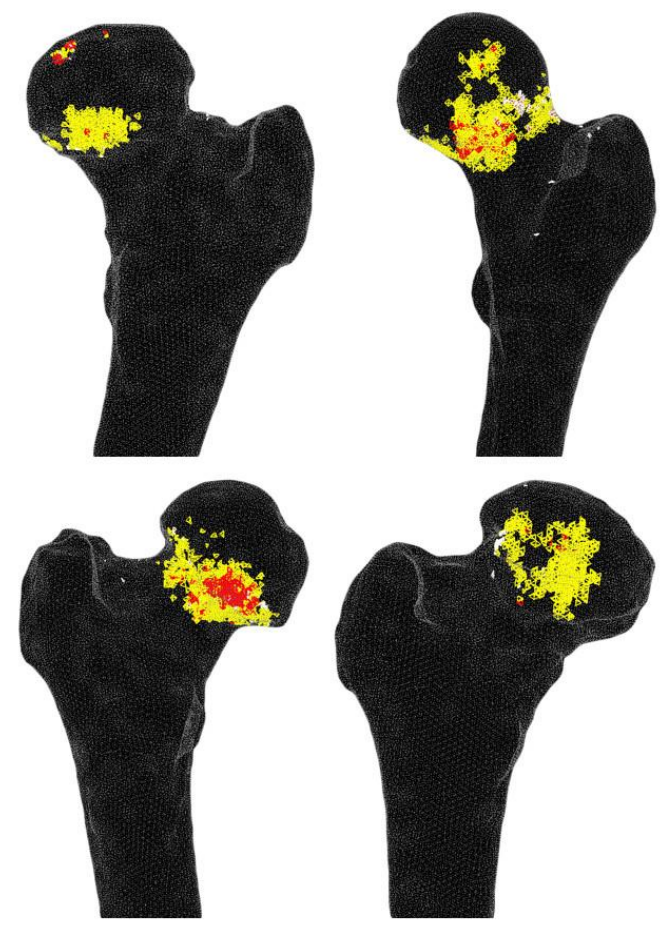

Fig.11: Estimated fracture locations of different osteoarthritis femurs.

\section{DISCUSSION}

Based on physical and mechanical properties, the normal femurs are apparently stronger than osteoarthritic ones. The superior distribution patterns of BMD, E, SED of the normal femur accompanied that they are about to be stronger than the osteoarthritis in which the patterns are randomly distributed. An overestimation of BMD and other properties might, therefore, happen in osteoarthritis femur.

The SED patterns of the normal femur are apparently consistent with that of a high BMD and Young's modulus distribution. The other researchers have also announced that the BMD pattern regulated by SED had the best similarity with the actual BMD [12,13]. Therefore, in this study, the calculated BMD values of the normal femurs can be in a state of reliability. The accumulation of failure elements in osteoarthritis femurs are obviously larger than the normal ones, and the ability to resist the applied load is lower as well.

Both types of femurs may fracture in the neck region. Nonetheless, the likelihood of fracture is more in osteoarthritis. Moreover, the fracture could also happen in the head of the osteoarthritis because of FAI involving both compressive and tensile failure. However, the neck fracture of the normal femur is principally associated with tensile failure, which agrees with the data reported by Ota et al. (1999) [14].

\section{CONCLUSION}

The results of the FEA study indicate that bone strength, fracture elements and fracture location of the proximal femur were affected by the severity of femoral head deformation. The estimation of femoral strength was found to be $3957 \pm 293 \mathrm{~N}$ and $3549 \pm 180 \mathrm{~N}$ for the normal and osteoarthritis femur, respectively. In general, the fracture loads of osteoarthritic femurs are noticeably lower than that of the normal ones. The fracture location of the normal femurs was determined to be at the neck region mostly. In osteoporosis, both the head and neck regions were estimated to be fractured.

Although a mechanical test on human femur was not unable to conduct in this study, the estimated neckcapital fractures in both femur types are in accord with another study, in which the authors reported that the experimental fracture site and fracture site through FEA are the same at the neck-capital region [15].

Furthermore, a great consistency between BMD and other mechanical properties, such as Young's modulus and SED, was well noted. Therefore, according to our data, it suggests that a better femoral strength determination can be obtained by considering not only BMD but other biomechanical properties as well. Moreover, the fracture elements in the FE model can be traced in detail by a sub-step loading system, in which how many elements fail in a single load step can be easily counted.

However, the sample size of the current study may be small, and the single-leg stance was barely analyzed. The majority of patient CT data were of women, and gender dependency was not studied yet. It might, therefore, have some limitations for the clinical generalization. Nonetheless, it is possible to estimate the femoral strength with reasonably high accuracy for a single-leg stance loading configuration with the patient-specific CT-based nonlinear FE method used in this study.

\section{ACKNOWLEDGMENTS}

The authors would like to thank AUN/SEED-Net and Japan International Cooperation Agency (JICA) for supporting research fund.

\section{REFERENCES}

[1] Sugano M, Hagiwara S, Nakamura J, Matsuura Y, Suzuki T, Wako Y, et al. Comparison study of bone strength of the proximal femur with and without hip osteoarthritis by computed tomography-based finite element analysis. J Biomech 2020;105:109810.

[2] Cody DD, Gross GJ, J. Hou F, Spencer HJ, Goldstein S a., P. Fyhrie D. Femoral strength is better predicted by finite element models than QCT and DXA. J Biomech 1999;32(10):1013-20.

[3] Keyak JH, Rossi SA, Jones KA, Skinner HB. Prediction of femoral fracture load using automated finite element modeling. J Biomech 1997;31(2):125-33.

[4] Keyak JH, Skinner HB. Three-dimensional finite element modelling of bone: effects of element size. J Biomed Eng 1992;14(6):483-9.

[5] Davis ET, Olsen M, Zdero R, Papini M, Waddell JP, Schemitsch EH. A biomechanical and finite element analysis of femoral neck notching during hip resurfacing. J Biomech Eng 2009;131(4):041002-8.

[6] Radcliffe IAJ, Taylor M. Investigation into the effect of varus-valgus orientation on load transfer in the resurfaced femoral head: A multi-femur finite element analysis. Clin Biomech 2007;22(7):780-6.

[7] Nor Aiman Nor Izmin, Todo M, Halim AA. Effects of Varus \& Valgus Implant Malposition in Resurfacing Hip Arthroplasty. In: International Exchange and Innovation Conference on 
Engineering \& Sciences. Kyushu University; 2019. page 89-92.

[8] Ulrich D, Van Rietbergen B, Weinans H, Rüegsegger P. Finite element analysis of trabecular bone structure: A comparison of imagebased meshing techniques. J Biomech 1998;31(12):1187-92.

[9] Dalstra M, Huiskes R, van Erning L. Development and validation of a three-dimensional finite element model of the pelvic bone. J Biomech Eng 1995;117(3):272-8.

[10] Keyak JH, Skinner HB, Fleming JA. Effect of force direction on femoral fracture load for two types of loading conditions. J Orthop Res 2001;19(4):539-44.

[11] Endo D, Ogami-Takamura K, Imamura T, Saiki K, Murai K, Okamoto K, et al. Reduced cortical bone thickness increases stress and strain in the female femoral diaphysis analyzed by a CT-based finite element method: Implications for the anatomical background of fatigue fracture of the femur. Bone Reports 2020;13:100733.

[12] Huiskes R, Rulmerman R, Van Lenthe GH, Janssen JD. Effects of mechanical forces on maintenance and adaptation of form in trabecular bone. Nature 2000;405(6787):704-6.

[13] Zhang Y, Luo Y. Femoral bone mineral density distribution is dominantly regulated by strain energy density in remodeling. Biomed Mater Eng 2020;31(3):179-90.

[14] Ota T, Yamamoto I, Morita R. Fracture simulation of the femoral bone using the finite-element method: How a fracture initiates and proceeds. J Bone Miner Metab 1999;17(2):108-12.

[15] Bessho M, Ohnishi I, Matsuyama J, Matsumoto T, Imai K, Nakamura K. Prediction of strength and strain of the proximal femur by a CT-based finite element method. J Biomech 2007;40(8):1745-53. 